\title{
Communication \\ Holographic Foam Cosmology: From the Late to the Early Universe
}

\author{
Yee Jack Ng
}

check for

updates

Citation: $\mathrm{Ng}$, Y.J. Holographic Foam Cosmology: From the Late to the Early Universe Symmetry 2021, 13, 435. https://doi.org/10.3390/ sym 13030435

Academic Editor: Chris Fields

Received: 19 February 2021

Accepted: 3 March 2021

Published: 8 March 2021

Publisher's Note: MDPI stays neutral with regard to jurisdictional claims in published maps and institutional affiliations.

Copyright: (C) 2021 by the author. Licensee MDPI, Basel, Switzerland. This article is an open access article distributed under the terms and conditions of the Creative Commons Attribution (CC BY) license (https:// creativecommons.org/licenses/by/ $4.0 /)$.
Department of Physics and Astronomy, Institute of Field Physics, University of North Carolina, Chapel Hill, NC 27599, USA; yjng@physics.unc.edu

\begin{abstract}
Quantum fluctuations endow spacetime with a foamy texture. The degree of foaminess is dictated by black hole physics to be of the holographic type. Applied to cosmology, the holographic foam model predicts the existence of dark energy with critical energy density in the current (late) universe, the quanta of which obey infinite statistics. Furthermore, we use the deep similarities between turbulence and the spacetime foam phase of strong quantum gravity to argue that the early universe was in a turbulent regime when it underwent a brief cosmic inflation with a "graceful" transition to a laminar regime. In this scenario, both the late and the early cosmic accelerations have their origins in spacetime foam.
\end{abstract}

Keywords: spacetime foam; holography; dark energy; cosmic inflation; infinite statistics; turbulence

\section{Introduction}

There are two cosmic accelerations that we are aware of: a brief inflationary acceleration [1-5] in the early universe and the present ("late" universe") acceleration [6,7] that is attributed to dark energy. Normally they are treated independently and separately; but there are also some works [8,9] that consider both regimes of accelerated expansions. We think it is conceptually necessary and aesthetically pleasing to trace both cosmic accelerations to a common cause in fundamental theory. Following Wheeler [10-12], we believe that space is composed of an ever-changing geometry and topology called spacetime foam and that the foaminess is due to quantum fluctuations of spacetime. We argue for a scenario in which spacetime foam is the origin of both cosmic accelerations.

The outline of this paper is as follows. We begin with a brief review of the holographic spacetime foam model. In Section 2, we use the quantum uncertainty principle coupled with black-hole physics to show that spacetime is indeed foamy and the degree of foaminess is consistent with the holographic principle; we further argue that there necessarily exists a dark sector in the universe. In Section 3, we apply the holographic spacetime foam to cosmology (with the corresponding cosmology called holographic foam cosmology (HFC) [13-16]) and argue for the existence of dark energy with critical energy density in the present universe and its quanta obey infinite statistics, also known as quantum Boltzmann statistics. In Section 4, we use the deep similarities between the physics of turbulence and the universal geometric properties of the holographic spacetime foam to heuristically argue that the early universe was in a turbulent phase during which the universe underwent a brief cosmic inflationary acceleration. Section 5 contains our concluding remarks, especially with respect to the naturalness and inevitability of inflation in the early universe.

We will use the subscript "P" to denote Planck units (with, e.g., $l_{P} \equiv\left(\hbar G / c^{3}\right)^{1 / 2} \sim$ $10^{-33} \mathrm{~cm}$ being the Planck length). Furthermore, for simplicity, $\hbar, c$, and the Boltzmann constant $k_{B}$ are often put equal to unity. 


\section{Holographic Spacetime Foam}

One manifestation of spacetime fluctuations is in the induced uncertainties in any distance measurement. Consider the following gedanken experiment [17] to measure the distance $l$ between a clock at one point and a mirror at another. By sending a light signal from the clock to the mirror in a timing experiment, we can determine the distance. The quantum uncertainty in the positions of the clock and the mirror introduces an inaccuracy $\delta l$. Let us concentrate on the clock (of mass $m$ ). If it has a linear spread $\delta l$ when the light signal leaves the clock, then its position spread grows to $\delta l+\hbar l(m c \delta l)^{-1}$ when the light signal returns to the clock, with the minimum uncertainty at $\delta l=(\hbar l / m c)^{1 / 2}$. Hence one concludes that $\delta l^{2} \gtrsim \frac{\hbar l}{m c}$. One can supplement this requirement with a limit from general relativity [18,19], viz., $\delta l$ must be larger than the Schwarzschild radius $G m / c^{2}$ of the clock, yielding $\delta l \gtrsim \frac{G m}{c^{2}}$ (henceforth we will neglect multiplicative constants of order unity), the product of which with the bound from quantum fluctuations finally gives $[18,20,21]$

$$
\delta l \gtrsim\left(l l_{P}^{2}\right)^{1 / 3}=l_{P}\left(\frac{l}{l_{P}}\right)^{1 / 3} .
$$

This bound on $\delta l$ can also be derived by the following method which provides additional valuable insights. Consider a spherical volume of radius $l$ over the amount of time $T=2 l / c$ it takes light to cross the volume. One way to map out the geometry of this spacetime region [22] is to fill the space with clocks, exchanging signals with other clocks and measuring the signals' times of arrival. This process of mapping the geometry is a sort of computation; hence the total number of operations is bounded by the Margolus-Levitin theorem [23], which stipulates that the rate of operations for any computer cannot exceed the amount of energy $E$ that is available for computation divided by $\pi \hbar / 2$. To avoid collapsing the region into a black hole, the total mass $M$ of clocks must be less than $l c^{2} / 2 G$, corresponding to the upper bound on energy density

$$
\rho \sim \frac{l / G}{l^{3}}=\left(l l_{P}\right)^{-2}
$$

Together, these two limits imply that the total number of operations that can occur in a spatial volume of radius $l$ for a time period $2 l / c$ is no greater than $\sim\left(l / l_{P}\right)^{2}$. (Here and henceforth we set $c=1=\hbar$ ). To maximize spatial resolution, each clock must tick only once during the entire time period. Furthermore, if we regard the operations partitioning the spacetime volume into "cells", then on the average each cell occupies a spatial volume no less than $\sim l^{3} /\left(l^{2} / l_{P}^{2}\right)=l l_{P}^{2}$, yielding an average separation between neighboring cells no less than $l^{1 / 3} l_{P}^{2 / 3}$. This spatial separation is interpreted as the average minimum uncertainty in the measurement of a distance $l$, that is, $\delta l \gtrsim l^{1 / 3} l_{P}^{2 / 3}$, in agreement with the result Equation (1) obtained above.

We can now heuristically derive the holographic principle. Since, on the average, each cell occupies a spatial volume of $(\delta l)^{3} \lesssim l l_{P}^{2}$, a spatial region of size $l$ can contain no more than $l^{3} /\left(l l_{P}^{2}\right)=\left(l / l_{P}\right)^{2}$ cells. Thus, this spacetime foam model corresponds to the case of maximum number of bits of information $l^{2} / l_{P}^{2}$ in a spatial region of size $l$, that is allowed by the holographic principle [24-27]. Accordingly, we will refer to this spacetime foam model (corresponding to $\delta l \gtrsim l^{1 / 3} l_{P}^{2 / 3}$ ) as the holographic spacetime foam model.

\section{From Spacetime Foam to Dark Energy}

As a corollary to the above discussion, we can now give a heuristic argument $[13,22,28]$ on why the universe cannot contain ordinary matter only. Start by assuming the universe (of size $l$ ) has only ordinary matter. According to the statistical mechanics for ordinary matter at temperature $T$, energy scales as $E \sim l^{3} T^{4}$ and entropy goes as $S \sim l^{3} T^{3}$. Black hole physics can be invoked to require $E \lesssim \frac{l}{G}=\frac{l}{l_{P}^{2}}$. Then it follows that the entropy $S$ and hence also the number of bits $I$ (or the number of degrees of freedom) on ordinary matter 
are bounded by $\lesssim\left(l / l_{P}\right)^{3 / 2}$. We can repeat verbatim the argument given in Section 2 to conclude that, if only ordinary matter exists, $\delta l \gtrsim\left(\frac{l^{3}}{\left(l / l_{P}\right)^{3 / 2}}\right)^{1 / 3}=l^{1 / 2} l_{P}^{1 / 2}$ which is much greater than $l^{1 / 3} l_{P}^{2 / 3}$, the result found above from our analysis of the gedanken experiment and implied by the holographic principle. Thus, there must be other kinds of matter/energy with which the universe can map out its spacetime geometry to a finer spatial accuracy than is possible with the use of only conventional ordinary matter. We conclude that a dark sector necessarily exists in the universe!

The above discussion leads to the prediction of dark energy. To see that, let us now generalize this discussion for a static spacetime region with low spatial curvature to the case of the recent/present universe by substituting $l$ by $1 / H$, where $H$ is the Hubble parameter $[13,28]$. Equation (2) yields the cosmic energy density $\rho \sim\left(\frac{H}{l_{P}}\right)^{2} \sim\left(R_{H} l_{P}\right)^{-2} \sim$ $10^{-120} M_{P}^{4}$ with $R_{H}$ being the Hubble radius. Next, recall that we have also shown that the universe contains $I \sim\left(R_{H} / l_{P}\right)^{2}$ bits of information $\left(\sim 10^{120}\right.$ for the current epoch) [13]. Hence the average energy carried by each of these bits or quanta is $\rho R_{H}^{3} / I \sim R_{H}^{-1}$. These long-wavelength bits or "particles" (quanta of spacetime foam) carry negligible kinetic energy. (Alternatively one can interpret these quanta as constituents of dark energy, contributing a more or less uniformly distributed cosmic energy density and hence acting as a dynamical effective cosmological constant $\Lambda \sim H^{2}$.) Note: Such long-wavelength quanta can hardly be called particles. We will simply call them "particles" in quotation marks. Since pressure (energy density) is given by kinetic energy minus (plus) potential energy, a negligible kinetic energy means that the pressure of the unconventional energy is roughly equal to minus its energy density, leading to accelerating cosmic expansion, in agreement with observation [6]. This scenario is very similar to that of quintessence [29,30], but it has its origin in the holographic spacetime foam [15,31].

How do these long-wavelength quanta differ from ordinary particles? Consider $N \sim\left(R_{H} / l_{P}\right)^{2}$ such "particles" in volume $V \sim R_{H}^{3}$ at $T \sim R_{H}^{-1}$, the average energy carried by each "particle". If these "particles" obey Boltzmann statistics, the partition function $Z_{N}=(N !)^{-1}\left(V / \lambda^{3}\right)^{N}$ gives the entropy of the system $S=N\left[\ln \left(V / N \lambda^{3}\right)+5 / 2\right]$, with thermal wavelength $\lambda \sim T^{-1} \sim R_{H}$. However, then $V \sim \lambda^{3}$, so $S$ becomes negative unless $N \sim 1$ which is equally nonsensical. A simple solution is to stipulate that the $N$ inside the $\log$ in $S$, i.e, the Gibbs factor $(N !)^{-1}$ in $Z_{N}$, must be absent. (This means that the $\mathrm{N}$ "particles" are distinguishable!) Then the entropy is positive: $S=N\left[\ln \left(V / \lambda^{3}\right)+3 / 2\right] \sim N$. Now, the only known consistent statistics in greater than 2 space dimensions without the Gibbs factor is the quantum Boltzmann statistics, also known as infinite statistics [32-34]. Thus, we conclude that the "particles" constituting dark energy obey infinite statistics, rather than the familiar Fermi or Bose statistics [28,35]. For completeness, let us list some of the properties of infinite statistics [32-34]. A Fock realization of infinite statistics is given by $a_{k} a_{l}^{\dagger}=\delta_{k, l}$. It is known that particles obeying infinite statistics are distinguishable, and importantly their theories are non-local [34,36] (to be more precise, the fields associated with infinite statistics are not local, neither in the sense that their observables commute at spacelike separation nor in the sense that their observables are pointlike functionals of the fields). Their quanta are extended (consistent with what we show above for dark energy). The number operator and Hamiltonian, etc., are both nonlocal and nonpolynomial in the field operators. This property of non-locality will be useful later in the discussion of the early universe. However, we should note that TCP theorem and cluster decomposition still hold; and quantum field theories with infinite statistics remain unitary [34].

\section{From Spacetime Foam and Turbulence to Cosmic Inflation}

To date, we have applied HFC to the present and recent cosmic eras (with $\rho \sim$ $10^{-120} M_{P}^{4}$ ). However, what about the early universe (with $\rho \sim 10^{-8} M_{P}^{4}$ )? Actually the discussion in the preceding section has already given us some helpful hints [13] especially with respect to inflation [1-5] in the early universe. For example: (1) The flatness problem is largely solved because, according to HFC, the cosmic energy is of critical density. 
(2) It is quite possible that HFC provides sufficient density perturbation as the model contains the essence of a k-essence model. (Furthermore, the horizon problem may also be solved since spacetime foam physics is essentially quantum black hole physics and thus is closely related to wormhole physics which can be used [37] to solve the horizon problem.) Nevertheless one important aspect of the early universe appears to be missing in HFC. It is connected to the expectation (supported by Wheeler's insight [38]) that, due to quantum fluctuations, spacetime, when probed at very small scales, as is the case for the early universe, will appear very complicated-something akin in complicity to a chaotic turbulent froth. So, was spacetime turbulent in the early universe? To this question we now sketch a positive response.

First let us show the deep similarities between the problem of quantum gravity and turbulence $[39,40]$. The connection between them can be traced to the role of diffeomorphism symmetry in classical gravity and the volume preserving diffeomorphisms of classical fluid dynamics. In the case of irrotational fluids in three spatial dimensions, the equation for the fluctuations of the velocity potential can be written in a geometric form [41] of a harmonic Laplace-Beltrami equation: $\frac{1}{\sqrt{-g}} \partial_{a}\left(\sqrt{-g} g{ }^{a b} \partial_{b} \varphi\right)=0$, where the effective space time metric has the canonical ADM form $d s^{2}=\frac{\rho_{0}}{c}\left[c^{2} d t^{2}-\delta_{i j}\left(d x^{i}-v^{i} d t\right)\left(d x^{j}-v^{j} d t\right)\right]$, with $c$ being the sound velocity. In this expression for the metric, it is apparent that the velocity of the fluid $v^{i}$ plays the role of the shift vector $N^{i}$ in the canonical Dirac/ADM treatment of Einstein gravity: $d s^{2}=N^{2} d t^{2}-h_{i j}\left(d x^{i}+N^{i} d t\right)\left(d x^{j}+N^{j} d t\right)$. Hence in the fluid dynamics context, $N^{i} \rightarrow v^{i}$, and a fluctuation of $v^{i}$ would imply a fluctuation of the shift vector (and hence a fluctuation of the spacetime metric) and vice versa.

Next, recall that the onset of turbulence can be predicted by the dimensionless Reynolds number $R e=L v / v$, where $v$ is the velocity field, $L$ is a characteristic scale and $v$ is the kinematic viscosity which is given by the product of the mean free path $\tilde{l}$ and an effective velocity factor $\tilde{v}$. At low Reynolds number, fluid flow is laminar, i.e., smooth; at high Reynolds number, the flow becomes turbulent. A characteristic signature for turbulence is the formation of eddies at various scales. The energy cascades from large-scale structures to smaller scale ones. The scale at which molecular diffusion becomes important and viscous dissipation of energy transpires is the Kolmogorov length which we will denote as $\ell$. Let us note that, in fully developed turbulence in three spatial dimensions, Kolmogorov scaling specifies the behavior of $n$-point correlation functions of the fluid velocity. The scaling [42,43] follows from the assumption of constant energy flux, $\frac{v^{2}}{t} \sim \varepsilon$, where $v$ stands for the velocity field of the flow, and the single length scale $\ell$ is given as $\ell \sim v \cdot t$. This implies that $v \sim(\varepsilon \ell)^{1 / 3}$, consistent with the experimentally observed two-point function $\left\langle v^{i}(\ell) v^{j}(0)\right\rangle \sim(\varepsilon \ell)^{2 / 3} \delta^{i j}$ (the famous two-thirds law, which yields, via a one-dimensional Fourier transformation, the energy scaling $E(k) \sim k^{-5 / 3}$, Kolmogorov's seminal " $5 / 3$ " law for the energy distribution in the turbulent fluid.) Now recall our discussion above on distance fluctuations $\delta \ell \sim \ell^{1 / 3} \ell_{P}^{2 / 3}$, and define the velocity as $v \sim \frac{\delta \ell}{t_{c}}$, since the natural characteristic time scale is $t_{c} \sim \frac{\ell_{p}}{c}$, then it follows that $v \sim c\left(\frac{\ell}{\ell_{p}}\right)^{1 / 3}$. (The implication is that at short distance, the spacetime is a chaotic and stochastic fluid in a turbulent regime with the Kolmogorov length $l$. The energy cascades are a property of the spacetime foam.) It is now obvious that a Kolmogorov-like scaling [42,43] in turbulence has been obtained. This interpretation of the Kolmogorov scaling in the quantum gravitational setting implies that the quantum fluctuation phase of strong quantum gravity in the early universe could be governed by turbulence.

The discussion above is for the case of very large Reynolds number. However, was $R e$ actually large enough in the early universe to set off turbulence? For the purpose of comparison and illustration, let us recall that, in conventional cosmology at time, say, $10^{-35} \mathrm{~s}, v L$ in the numerator of $R e$ is roughly given by the product of $v \sim 10^{-2} \mathrm{c}$ and $L \sim 10^{8} l_{P}$ [44-46]. For HFC close to Planckian time, the denominator of $R e$ is given by $v \sim$ $c l_{P}$ since, in that regime, momentum transport could only be due to Planckian dynamics. For the discussion to follow, let us note that, relatively speaking, the effective velocity factor 
$\tilde{v}$ in $v$ is not that different from the $v$ factor in the numerator of $R e$. The onset of turbulence was due to the smallness of the length scale (which plays the role of an effective mean free path) in the denominator of $R e$, viz., $\tilde{l}=l_{P}<<L \sim 10^{8} l_{P}$ that was mainly responsible for yielding a large $R e$ in the (very) early universe.

There remains one crucial hurdle to overcome. If, as we suggest, turbulence in the early universe was related to inflation, we have to confront the "graceful" exit problem: How to get a small enough $R e$ to transit to the laminar phase and to end inflation in the process? It is here the nonlocality property enjoyed by the quanta of spacetime foam (due to the fact that they obey infinite statistics) came to the rescue since the length scale $\tilde{l}$ in $v$ would naturally and eventually extend to the order of $L$. (Compare with the case of dark energy discussed in Section 3.) This would yield a small enough Re to suppress turbulence and to naturally end inflation.

\section{Conclusions}

We have sketched a scenario in which both the late and the early cosmic accelerations have a common origin and can be traced to spacetime foam. The case for dark energy in the current/recent ("late") universe was proposed before $[13,28]$, while the case for cosmic inflation in the early Univerese is the main focus of this paper. One attractive feature of our present proposal is that the scheme is very economical, involving no arbitrary or fine-tuned parameters. It is also natural in that inflation was inevitable as turbulence set off by the Planckian dynamics was inevitable in the early universe. The scheme also provides a rationale for why inflation lasted only briefly (say, $\sim 10^{-33} \mathrm{~s}$ ) as the turbulent phase was quickly terminated due to the nonlocal (extended) property of the quanta of spacetime foam. Of course it is important to check if this scenario is supported by more quantitative arguments and calculations. In passing we should also mention that it will be of great interest to see if our scenario for inflation discussed above can at least mitigate some of the criticism $[47,48]$ against the inflation paradigm.

We conclude with two observations the first of which involves the crucial question of whether our approach yields enough e-folds of inflation to solve the myriad of cosmological problems. The following heuristic argument would seem to say probably there were. Let us follow the folklore: at the end of inflation, the energy stored in the quanta of spacetime foam would be converted into hot ordinary particles (as well as dark matter). Since the Grand Unification era is around $\sim 10^{-34} \mathrm{~s}$, which, as an order-of-magnitude estimate, should also mark the end of inflation, giving enough (say $\gtrsim 65$ ) e-folds of inflation. This argument may be strengthened, if, as has been proposed [49], quantum gravity can actually be the origin of (ordinary-) particle statistics, and that infinite statistics (the statistics obeyed by quanta of spacetime foam) is the underlying statistics. In that case, ordinary particles that obey Bose or Fermi statistics are actually some sort of collective degrees of freedom of "particles" of infinite statistics. (See [50] for a discussion of such a construction.) Thus, arguably, at the end of inflation, quanta of spacetime foam could be converted into ordinary particles as required.

We end this paper with our second observation. Our aesthetically pleasing scenario can be compared to a recent model [8] of quintessential inflation based on the assumption that the slow roll parameter has a Lorentzian form as a function of the number of e-folds. Its form corresponds to the vacuum energy both in the inflationary (with $\rho \sim 10^{-8} M_{P}^{4}$ ) and the dark energy (with $\rho \sim 10^{-120} M_{P}^{4}$ ) epochs which are treated symmetrically. In this model the inflationary scale is exponentially amplified while the dark energy scale is suppressed, producing a curious cosmological see-saw mechanism. In the present work, the two cosmic accelerations are also attributed to a single mechanism; but they are related by some sort of turbulent-laminar duality (or chaotic-smooth complementarity). It would be interesting to see if our scheme can be approximated by an effective theory in which a similar ansatz for the slow roll parameter naturally arises as in the see-saw model [8]. However, a full investigation may require a non-perturbative treatment of quantum gravity, involving a truly non-local field theory of "particles" obeying infinite statistics. (We note 
that when the see-saw model is realized in the context of a single scalar field, the extracted potential of the scalar field is fairly complicated. Can this complication be a reflection of non-perturbative quantum gravity involving non-local quanta of spacetime foam?)

Funding: This research was partly supported by the Bahnson Fund and the Kenan Professors Research Fund of the University of North Carolina at Chapel Hill.

Institutional Review Board Statement: Not applicable.

Informed Consent Statement: Not applicable.

Data Availability Statement: Not applicable.

Acknowledgments: I thank David Benisty and Eduardo Guendelman for a useful correspondence. I am grateful to the Bahnson Fund and the Kenan Professors Research Fund of the University of North Carolina at Chapel Hill for partial financial support.

Conflicts of Interest: The author declares no conflict of interest.

\section{References}

1. Guth, A.H. Inflationary universe: A possible solution to the horizon and flatness problems. Phys. Rev. D 1981, 23, 347-356. [CrossRef]

2. Starobinsky, A.A. A new type of isotropic cosmological models without singularity. Phys. Lett. B 1980, 91, 99-102. [CrossRef]

3. Kazanas, D. Dynamics of the universe and spontaneous symmetry breaking. Astrophys. J. 1980, 241, L59-L63. [CrossRef]

4. Linde, A.D. A new inflationary universe scenario: A possible solution of the horizon, flatness, homogeneity, isotropy and primordial monopole problems. Phys. Lett. B 1982, 108, 389-393. [CrossRef]

5. Albrecht, A.; Steinhardt, P.J. Cosmology for grand unified theories with radiatively induced symmetry breaking. Phys. Rev. Lett. 1982, 48, 1220-1223. [CrossRef]

6. Perlmutter, S.; Aldering, G.; Goldhaber, G.; Knop, R.A.; Nugent, P.; Castro, P.G.; Deustua, S.; Fabbro, S.; Goobar, A.; Groom, D.E.; et al. Measurements of Omega and Lambda from 42 High-Redshift Supernovae. Astrophys. J. 1999, 517, 565-586. [CrossRef]

7. Riess, A.G.; Filippenko, A.V.; Challis, P.; Clocchiatti, A.; Diercks, A.; Garnavich, P.M.; Gilliland, R.L.; Hogan, C.J.; Jha, S.; Kirshner, R.P.; et al. Observational Evidence from Supernovae for an Accelerating Universe and a Cosmological Constant. Astron. J. 1998, 116, 1009-1038. [CrossRef]

8. Benisty, D.; Guendelman, E.I. Lorentzian Quintessential Inflation. Int. J. Mod. Phys. D 2020, 29, 2042002. [CrossRef]

9. Garcia-Bellido, J.; Rubio, J.; Shaposhnikov, M.; Zenhausern, D. Higgs-Dilaton Cosmology: From the Early to the Late Universe. Phys. Rev. D 2011, 84, 123504. [CrossRef]

10. Wheeler, J.A. Relativity, Groups and Topology; DeWitt, B.S., DeWitt, C.M., Eds.; Gordon \& Breach: New York, NY, USA, $1963 ;$ p. 315.

11. Hawking, S.W.; Page, D.N.; Pope, C.N. Quantum Gravitational Bubbles. Nucl. Phys. 1980, 170, 283-306. [CrossRef]

12. Ashtekar, A.; Rovelli, C.; Smolin, L. Weaving a Classical Geometry with Quantum Threads. Phys. Rev. Lett. 1992, 69, 237-240. [CrossRef] [PubMed]

13. Arzano, M.; Kephart, T.W.; Ng, Y.J. From Spacetime Foam to Holographic Foam Cosmology. Phys. Lett. B 2007, 649, 243. [CrossRef]

14. Maziashvili, M. Space-Time in Light of Karolyhazy Uncertainty Relation. Int. J. Mod. Phys. D 2007, 16, 1531-1539. [CrossRef]

15. Fischler, W.; Susskind, L. Holography and Cosmology. arXiv 1998, arXiv:hep-th/9806039.

16. Easther, R.; Lowe, D. Holography, Cosmology, and the Second Law of Thermodynamics. Phys. Rev. Lett. 1999, 82, 4967-4970. [CrossRef]

17. Salecker, H.; Wigner, E.P. Quantum Limitations of the Measurement of Space-Time Distances. Phys. Rev. 1958, 109, 571-577. [CrossRef]

18. Ng, Y.J.; van Dam, H. Limit to Spacetime Measurement. Mod. Phys. Lett. A 1994, 9, 335-340.

19. Ng, Y.J.; van Dam, H. Remarks on Gravitational Sources. Mod. Phys. Lett. A 1995, 10, 2801-2808. [CrossRef]

20. Karolyhazy, F. Gravitation and Quantum Mechanics of Macroscopic Objects. Il Nuovo Cimento A 1966, 42, 390-402. [CrossRef]

21. Sasakura, N. An Uncertainty Relation of Space-Time. Prog. Theor. Phys. 1999, 102, 169-179. [CrossRef]

22. Lloyd, S.; Ng, Y.J. Black Hole Computers. Sci. Am. 2004, 291, 52-61. [CrossRef] [PubMed]

23. Margolus, N.; Levitin, L.B. The Maximum Speed of Dynamical Evolution. Phys. D 1998, 120, 188-195. [CrossRef]

24. Hooft, G. Salamfestschrift; Ali, G.A., Ed.; World Scientific: Singapore, 1993; p. 284.

25. Susskind, L. The World as a Hologram. J. Math. Phys. 1995, 36, 6377-6396. [CrossRef]

26. Maldacena, J. The large N limit of superconformal field theories and supergravity. Adv. Theor. Math. Phys. 1998, 2, 231-252. [CrossRef]

27. Gambini, R.; Pullin, J. Holography in Spherically Symmetric Loop Quantum Gravity. Int. J. Mod. Phys. D 2008, 17, 545-549. [CrossRef]

28. Ng, Y.J. Holographic Foam, Dark Energy and Infinite Statistics. Phys. Lett. B 2007, 657, 10-14. [CrossRef] 
29. Ratra, B.; Peebles, J. Cosmological consequences of a rolling homogeneous scalar field. Phys. Rev. D 1988, 37, 3406-3427. [CrossRef]

30. Caldwell, R.R.; Dave, R.; Steinhardt, P.J. Cosmological Imprint of an Energy Component with General Equation-of-State. Phys. Rev. Lett. 1998, 80, 1582. [CrossRef]

31. Ng, Y.J. Proceedings of the Fortieth Karpacz Winter School on Theoretical Physics; Kowalski-Glikman, J., Amelino-Camelia, G., Eds.; Springer: Berlin, Germany, 2005; p. 321.

32. Doplicher, S.; Haag, R.; Roberts, J. Local Observables and Particle Statistics I. Commun. Math. Phys. 1971, 23, 199-230. [CrossRef]

33. Doplicher, S.; Haag, R.; Roberts, J. Local Observables and Particle Statistics II. Commun. Math. Phys. 1974, 35, 49-85. [CrossRef]

34. Greenberg, O.W. Example of Infinite Statistics. Phys. Rev. Lett. 1990, 64, 705-708. [CrossRef]

35. Jejjala, V.; Kavic, M.; Minic, D. Fine Structure of Dark Energy and New Physics. Adv. High Energy Phys. 2007, $2007,21586$. [CrossRef]

36. Fredenhagen, K. On the Existence of Antiparticles. Commun. Math. Phys. 1981, 79, 141-151. [CrossRef]

37. Hochberg, D.; Kephart, T.W. Wormhole Cosmology and the Horizon Problem. Phys. Rev. Lett. 1993, 70, 2665. [CrossRef] [PubMed]

38. Wheeler, J.A. Geons. Phys. Rev. 1955, 97, 511-536. [CrossRef]

39. Jejjala, V.; Minic, D.; Ng, Y.J.; Tze, C.H. Turbulence and holography. Class. Quant. Grav. 2008, 25, 225012. [CrossRef]

40. Jejjala, V.; Minic, D.; Ng, Y.J.; Tze, C.H. Quantum gravity and turbulence. Int. J. Mod. Phys. D 2010, 19, 2311-2317. [CrossRef]

41. Unruh, W. Dumb holes and the effects of high frequencies on black hole evaporation. Phys. Rev. D 1995, 51, 2827-2838. [CrossRef] [PubMed]

42. Kolmogorov, A.N. The local structure of turbulence in incompressible viscous fluid for very large Reynolds number. Dokl. Akad. Nauk SSSR 1941, 30, 299-303.

43. Kolmogorov, A.N. Dissipation of energy in the locally isotropic turbulence. Dokl. Akad. Nauk SSSR 1941, 32, 16-18.

44. Gibson, C.H. The First Turbulent Combustion. Combust. Sci. Tech. 2005, 177, 1. [CrossRef]

45. Krymsky, A.M.; Marochnik, L.S.; Naselsky, P.D.; Pelikhov, N.V. Turbulence in Cosmology III. The effect of non-linear interactions on the Universe expansion law, Quantum turbulence near singularity. Astrophys. Space Sci. 1978, 55, 325-350. [CrossRef]

46. Huang, K.; Low, H.B.; Tung, R.S. Scalar Field Cosmology II: Superfluidity, Quantum Turbulence, and Inflation. Int. J. Mod. Phys. A 2012, 27, 1250154. [CrossRef]

47. Penrose, R. Difficulties with Inflationary Cosmology. Ann. N. Y. Acad. Sci. 1989, 271, 249-264. [CrossRef]

48. Steinhardt, P.J. The inflation debate: Is the theory at the heart of modern cosmology deeply flawed? Sci. Am. 2011, 304, 36-43. [CrossRef]

49. Ho, C.M.; Minic, D.; Ng, Y.J. Dark matter, infinite statistics, and quantum gravity. Phys. Rev. D 2012, 85, 104033. [CrossRef]

50. Greenberg, O.W.; Delgado, J.D. Construction of bosons and fermions out of quons. Phys. Lett. A 2001, 288, 139. [CrossRef] 